\title{
FEATURE
}

\section{Light Microscopy Subject Guide}

\author{
(c) ASM International 2010
}

Editor's Note ASM International offers on its website a number of ASM Subject Guides. These provide a broad overview to selected topics including basic terminology, additional resources, article databases, catalogs, background information, web sites, and more. These pages were created by subject area specialists who work with the materials or process listed.

The ASM Subject Guide on Light Microscopy is included here for the benefit of the readers of Metallography, Microstructure, and Analysis.

Additional Subject Guides currently available include

- Aluminum and Aluminum Alloys

- Copper and Copper Alloys

- Corrosion

- Crystalline Structure

- Failure Analysis

- Heat Treating

- Phase Diagrams

- Shape Memory Effect, Superelasticity, and Shape Memory Alloys

- Stainless Steels

- Superalloys

- Titanium and Titanium Alloys

To suggest additional Subject Guides or volunteer to create one, contact Scott Henry, ASM International, 440-3385151, ext. 5706; email: scott.henry@asminternational.org.

\section{Introduction}

The light microscope remains the most important tool for the study of microstructure, despite the evolution of sophisticated electron microscopes. For example, reflected light microscopy is common in the imaging of metallic microstructures, while transmitted light microscopy can be more effectively used in the microstructural imaging of polymers. In general, all nonmetallic materials are suitable for examination by transmission polarized light microscopy. The choice of method also depends on imaging requirements such as resolution, magnification, depth of field, and lens aberration. These factors may influence the choice of light or electron microscopy. Scanning electron microscopy and transmission electron microscopy are invaluable techniques as well; however, they should be used in conjunction with light microscopy, rather than a substitute.

Video cameras have become ubiquitous in light microscopy labs-they are relatively inexpensive, can capture movement, offer good color quality, and are compatible with TV monitors, VCRs and DVDs, allowing easy display and recording. However, they are restricted to very low resolutions due to limitations of the standards.

Light microscopes can be classified as "upright" or "inverted"; these terms refer to the orientation of the light path to plane-of-polish of the specimen during observation (Fig. 1). Because each configuration has certain advantages and disadvantages, selection generally is based on personal preference.

The simplest light microscope is the bench type (usually upright, Fig. 2). Photographic capabilities may include digital recording attachments or a bellows for the connection of a camera, depending on the rigidity of the stand and the size and weight of the camera.

\section{Illumination Systems}

The illumination system for incident light microscopy consists of the lamp, lenses, filters, and diaphragms that are 


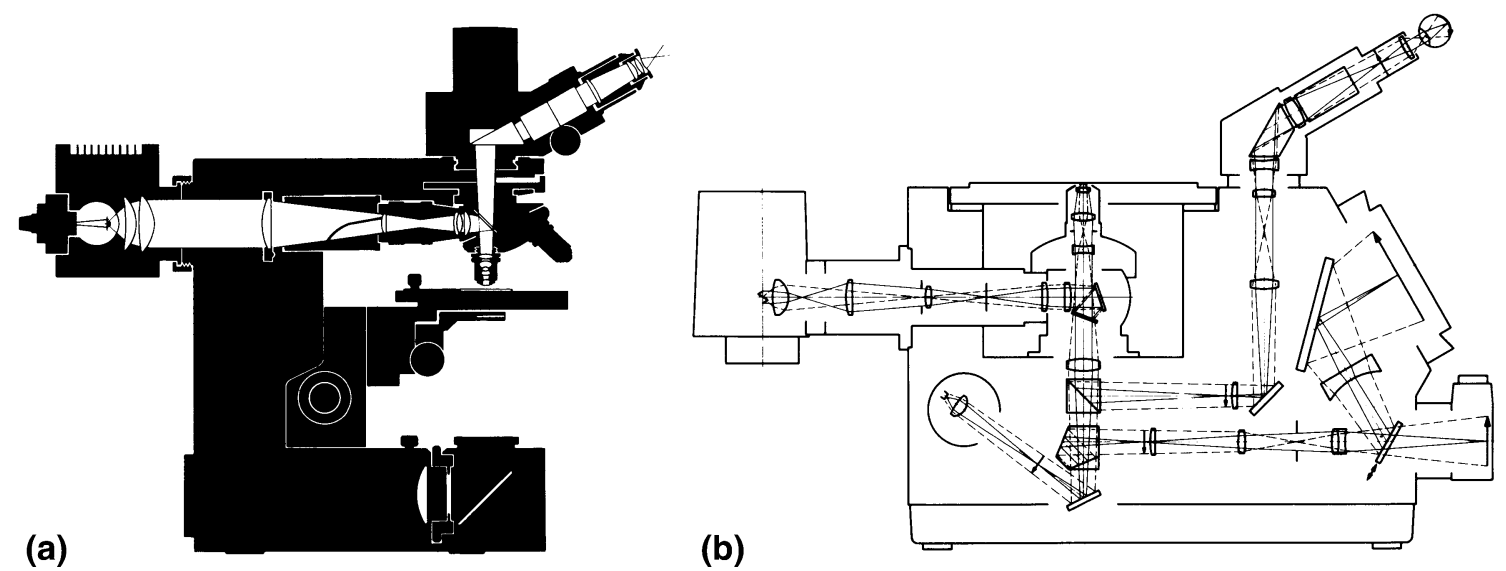

Fig. 1 Light paths in (a) an upright incident-light microscope and (b) an inverted incident-light microscope. Source Ref 1

Fig. 2 (a) Upright and (b) inverted bench-type microscopes. Courtesy of Carl Zeiss, Inc. and Leica, Inc. Source Ref 1
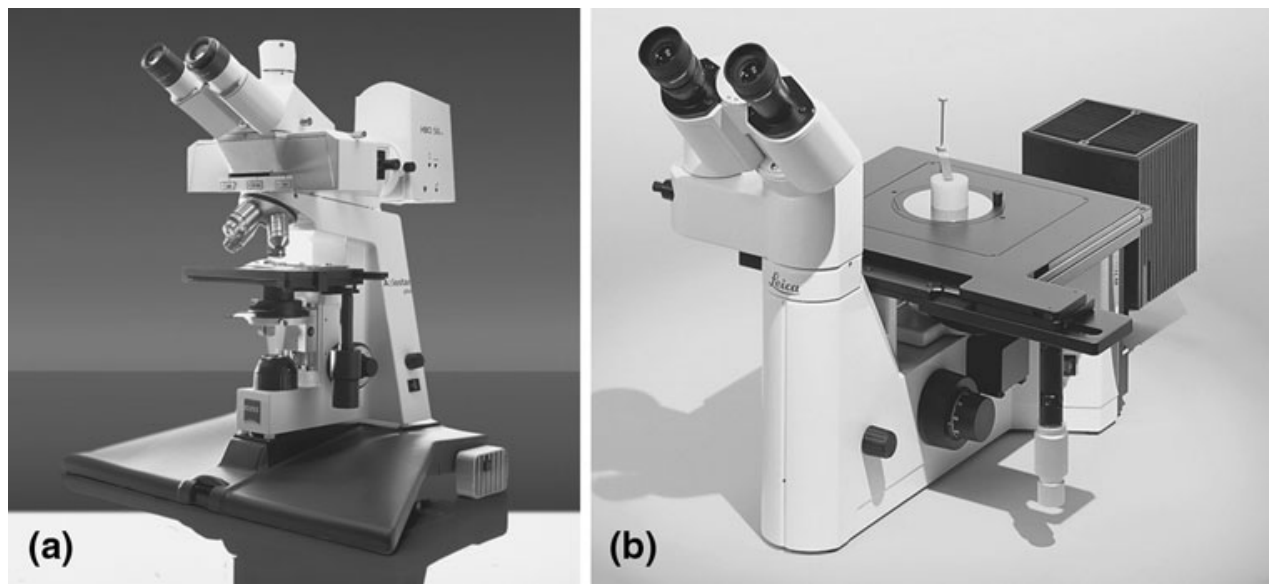

along the light path between the light source and the specimen. Generally, a uniformly illuminated object field exactly the size of the field of view is desired. The light should be adjustable in intensity, color, and polarization.

Reflected light microscopes apply special illumination techniques to reveal details of the microstructure. These optical techniques include dark-field illumination, polarized light microscopy, phase contrast microscopy, and differential interference contrast, all of which use the Köhler illumination principle to provide uniform illumination of the micro-section. A uniform light field at the object is desired, but the light source itself is non-uniform.

The Köhler principle uses the collector lens to form an image of the light source at the first condenser lens or at the illumination condenser aperture (Fig. 3). The second condenser lens reproduces the image of the light source in the back focal plane of the objective lens after reflection of the light at the reflector (plane glass, half-silvered mirror, or prism). Therefore, the surface of the specimen is uniformly illuminated. The condenser lenses and the objective form an image of the radiant field stop in the plane of the specimen surface.

\section{Light Sources}

A variety of light sources are available for light microscopy. The low-voltage tungsten-filament lamp is used primarily with bench microscopes. The light intensity can be varied by controlling the power to the lamp. The xenonarc light source is prevalent in older instruments (pre1980s) because of its high intensity and daylight color of its emission spectra characteristics. Light intensity, however, can be adjusted only by the use of neutral-density filters.

Tungsten-halogen filament lamps (usually $100 \mathrm{~W}$ ) are widely used for their high intensity and high color temperature (temperature in degrees Kelvin necessary to provide a color equivalent to that of the source in question). Light intensity can be controlled by varying the current or by use of neutral-density filters.

\section{Optical Performance}

Image aberrations are a consequence of the laws of reflection and refraction. Aberrations may be due to defects 
Fig. 3 The Kohler illumination principle in incident light microscopy. Source Ref 1

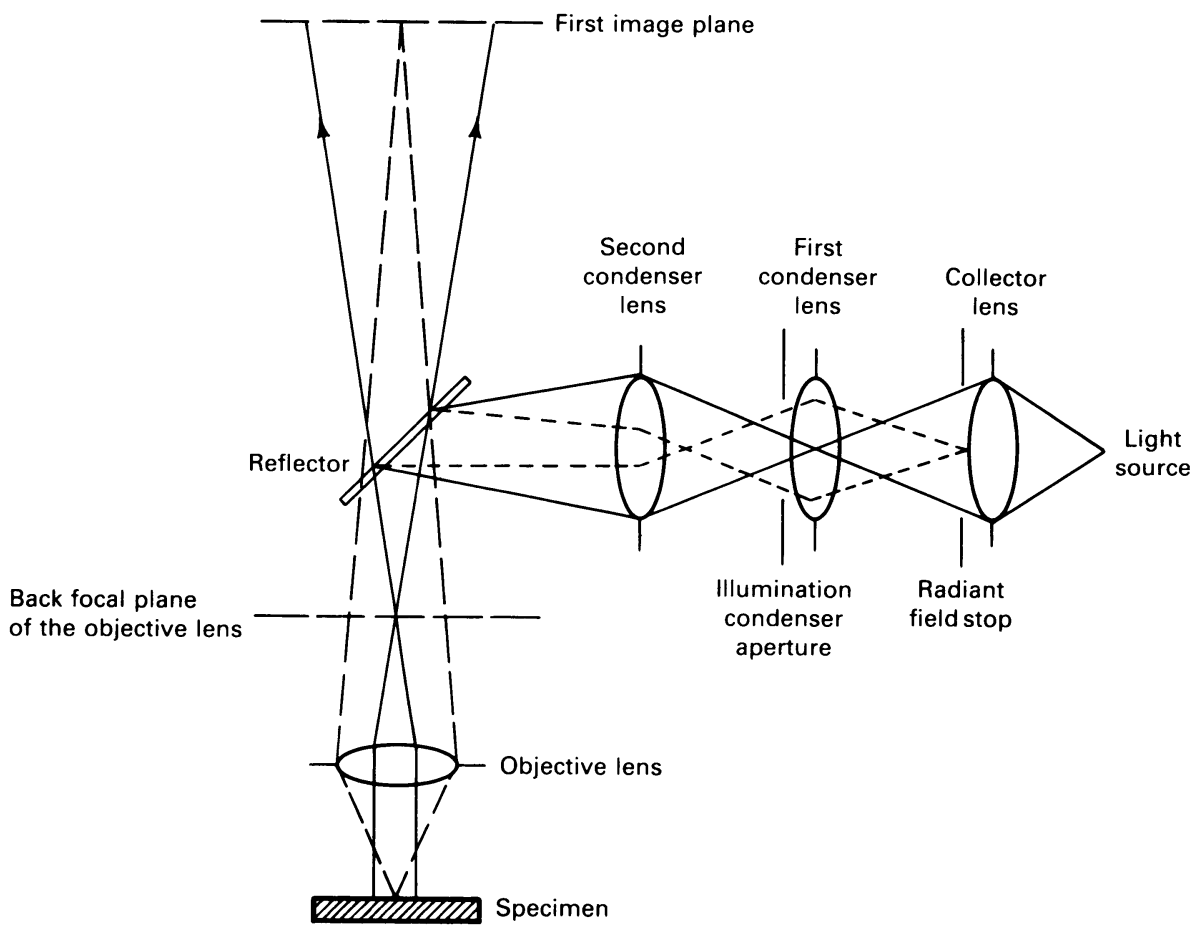

or to the inherent limitations of an optical system. Types of aberrations include:

- Astigmatism

- Coma

- Distortion

- Chromatic aberration

- Lateral color

- Curvature of field

- Spherical aberration

It is impossible to completely remove all the source aberrations with an optical system, and removing or reducing aberrations can increase the complexity and costs of a lens systems.

\section{Optical Components}

The objective lens forms the primary image of the microstructure and is the most important component of the light microscope. It is the lens closest to the object of interest. It collects as much light as possible from the specimen and combines this light to produce the image.

The condenser is an adjustable lens placed in front of the light source to focus the light at the desired point in the optical path. A field diaphragm is placed in front of this lens to minimize internal glare and reflections within the microscope. The field diaphragm is stopped down to the edge of the field of view, so the size of the illuminated area is limited to the observed field to minimize stray light.
The vertical illuminator usually provides only one or two types of illumination, such as bright-field and darkfield illumination, or bright-field and polarized light illumination. However, universal vertical illuminators are available that provide all types of illumination with one vertical illuminator and one set of objectives. Modern light microscopes are based on the principle of infinity corrected optics. This means that the reflected light forms a parallel beam between the objective and the tube lens. This principle allows placement of reflectors, prisms, and other components in the vertical illuminator without altering the magnification or the formation of the secondary image in the eyepieces.

The tube length is the length of the body tube from the eye line of the eyepiece to the objective thread. Most objectives are designed for use with a certain tube length, generally $160-250 \mathrm{~mm}(6-10 \mathrm{in}$.) and generally cannot be interchanged without changing the total magnification of the optical system.

The most commonly used objective is the achromat, which is corrected for spherical aberration for one color (usually yellow-green) and for longitudinal chromatic aberration for two colors (usually red and green). Therefore, achromats are not suitable for color photomicroscopy, except at low magnifications.

The parfocal distance of the objective is an important limiting factor for working distance. Working distance decreases as magnification of the objective increases. With parfocal lens systems, each objective on the nosepiece turret will be nearly in focus when the turret is rotated, 
preventing the objective front lens from striking the specimen when lenses are switched.

Certain objectives are designed for use with oil between the specimen and the front lens of the objective. Oil-immersion lenses are rarely used, because the specimen and lens must be cleaned after use. However, they do provide higher resolutions than can be achieved when air is between the lens and specimen. Use of oil also increases contrast and reducing glare of the image, which is valuable when examining lowreflectivity specimens, such as polymers or ceramics.

The eyepiece, or ocular, magnifies the primary image produced by the objective; the eye can then use the full resolution capability of the objective. The microscope produces a virtual image of the specimen at the point of most distinct vision, generally $250 \mathrm{~mm}$ (10 in.) from the eye. The eyepiece magnifies this image, permitting achievement of useful magnifications. The standard eyepiece has a $24 \mathrm{~mm}$ diameter field of view.

A $\times 10$ magnification eyepiece is usually used; to obtain standard magnifications, some systems require other magnifications, such as $\times 6.3$. The overall magnification is found by multiplying the objective magnification by the eyepiece magnification. The upper limit of useful magnification in a light microscope is approximately $\times 1500$, and the fundamental limitations of light optic systems limit resolution to features of $\sim 0.2 \mu \mathrm{m}$ or larger.

Eye clearance is the distance between the eye lens of the ocular and the eye. For most eyepieces, the eye clearance is $10 \mathrm{~mm}$ or less-inadequate if the microscopist wears glasses. Simple vision problems, such as nearsightedness, can be accommodated using the fine focus adjustment.

A second adjustable-iris diaphragm, the aperture diaphragm, is placed in the light path before the vertical illuminator. Opening or closing this diaphragm alters the amount and angle of light entering the objective lens. Opening this aperture increases image resolution, but reduces contrast; closing the aperture increases contrast, but diminishes image resolution.

Light filters are used to modify the light for ease of observation, for improved photomicroscopy, or to alter contrast. Neutral-density filters are used to reduce the light intensity uniformly across the visible spectrum. Most light microscopes offer at least two such filters.

Selective filters are used to balance the color temperature of the light source to that of the film. This is often necessary for faithful reproduction of color images, depending on the light source used and the film type. Most objectives require such filtering for best results, as these objectives are not fully corrected for chromatic aberrations.

An example of a microstructure revealed through optical microscopy of a common titanium base alloy is shown in Fig. 4.

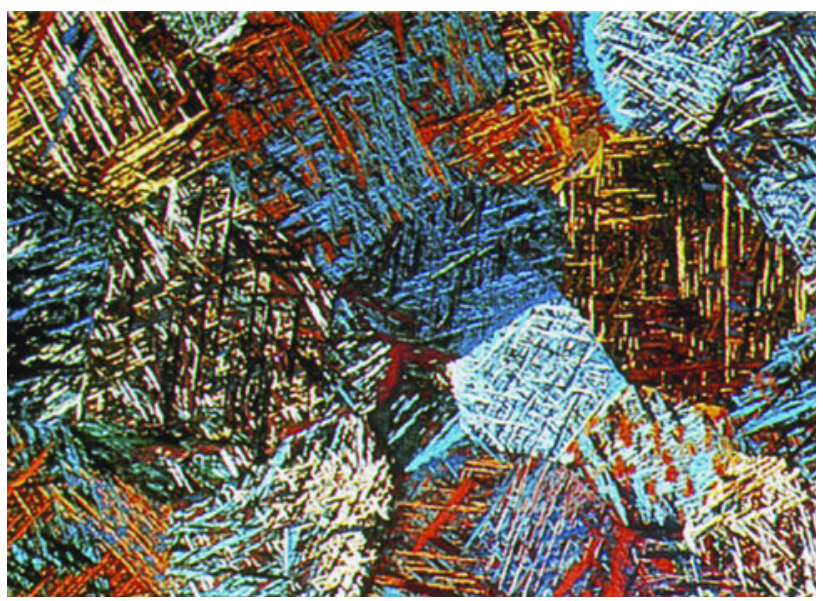

Fig. $4 \mathrm{Ti}-6 \mathrm{Al}-4 \mathrm{~V}$ alloy containing martensite needles formed at elevated temperature $\left(>840{ }^{\circ} \mathrm{C}\right.$ or $\left.1540{ }^{\circ} \mathrm{F}\right)$. Color etched in $100 \mathrm{~mL}$ $\mathrm{H}_{2} \mathrm{O}, 4 \mathrm{~mL} \mathrm{HCl}, 3 \mathrm{~g} \mathrm{~N} \mathrm{H}_{4} \mathrm{HF}_{2}$. Polarized light illumination. Original magnification: $\times 100$. Courtesy of G. Möller. Source Ref 1

\section{Examination Modes}

To achieve the resolution capability of the selected objective, image contrast must be adequate. Image contrast depends on specimen preparation and optics. Differences in light reflectivity from the specimen surface produce amplitude features visible to the eye after magnification.

Bright-Field Illumination. Bright-field vertical illumination is the most widely used method of observation. Light passes through the objective and strikes the specimen surface perpendicularly. Surface features normal to the incident light reflect light back through the objective to the eyepieces, where the surface features appear bright. Surfaces oblique to the light beam reflect less light and appear darker, depending on their angle.

Oblique Illumination. With some microscopes, it is possible to decenter the condenser assembly or the mirror so that the light passing through the objective strikes the specimen surface at a non-perpendicular angle. Roughness on the specimen surface will cast shadows, producing a three-dimensional appearance. This allows determination of features that are in relief or are recessed.

Dark-Field Illumination. The method of dark-field illumination is based on the effect of diffraction contrast, whereby light hitting the edge of an object bends and is diffracted out of the optical path. Only those light rays deflected by diffuse scattering from their original direction toward the optical axis of the microscope are used for image formation. The light reflected from obliquely oriented features is collected, and the rays reflected from features normal to the incident beam are blocked. Therefore, surface regions perpendicular to the optical axis will appear dark, and angled surfaces will appear light. 
There are three basic ways in which an image can be formed. Perhaps the simplest to imagine is the projection image; the most common example is the formation of shadows when an object is placed in front of a point source of illumination. The second type of image is formed by conventional lens systems, called an optical image. This is not a strictly accurate term, because optical often refers to imaging systems involving light. However, the term optical also is sometimes used in the general sense of images formed using light electrons or ions.

Both projection and optical images are formed in parallel; that is, all parts of the image are formed essentially simultaneously. However, a third type of image is the scanning image, in which each point of the picture is presented serially. With this type of image, several thousand picture points are displayed consecutively, but the process is repeated with such a high frequency that the image appears to the eye in its entirely.

\section{Depth of Field and Depth of Focus}

In any microscope, the image is only accurately in focus when the object lies in the appropriate plane. If part of the object being viewed lies above or below this plane, then the equivalent part of the image will be out of focus. The range of positions for the object for which our eye can detect no change in the sharpness of the image is known as the depth of field. In most microscopes, this distance is rather small; therefore, in order to produce sharp images, the object must be very flat. If a non-flat object (or a transparent object of appreciable thickness) is viewed at high magnification using a light microscope, then some out-of-focus regions will be seen. This is a useful feature for accentuating certain parts of the image at the expense of others but is a grave disadvantage for seeing all parts of a threedimensional object clearly.

\section{Reference}

1. ASM Handbook, Metallography and Microstructures, vol. 9 (ASM International, Materials Park, 2004)

\section{Selected References}

B.L. Bramfitt, A.O. Benscoter, Metallographer's Guide: Practices and Procedures for Irons and Steels (ASM International, Materials Park, 2002)

R.E. Chinn, Ceramography: Preparation and Analysis of Ceramic Microstructures (ASM International, Materials Park, 2002)

M. Gauthier (ed.), Engineered Materials Handbook, Desk edn. (ASM International, Materials Park, 1995)

B.S. Hayes, L.M. Gammon, Optical Microscopy of Fiber-Reinforced Composites (ASM International, Materials Park, 2010)

G. Petzow, Metallographic Etching, 2nd edn. (ASM International, Materials Park, 1999)

L.E. Samuels, Light Microscopy of Carbon Steels (ASM International, Materials Park, 1999)

G.F. Vander Voort, Metallography: Principles and Practice (ASM International, Materials Park, 1984) 